\section{$\underset{\substack{\text { hommes } \\ \text { \& migrations }}}{ }$}

\section{Hommes \& migrations}

Revue française de référence sur les dynamiques

migratoires

$1293 \mid 2011$

L'immigration dans les musées

\title{
L'oubli au cœur de la mémoire
}

Le Museo Nacional de la Inmigración d'Argentine

\section{Tamar Blickstein}

\section{(2) OpenEdition}

1 Journals

\section{Édition électronique}

URL : http://journals.openedition.org/hommesmigrations/515

DOI : 10.4000/hommesmigrations.515

ISSN : 2262-3353

Éditeur

Musée national de l'histoire de l'immigration

\section{Édition imprimée}

Date de publication : 1 septembre 2011

Pagination : 96-106

ISSN : 1142-852X

\section{Référence électronique}

Tamar Blickstein, «L'oubli au cœur de la mémoire », Hommes \& migrations [En ligne], 1293 | 2011, mis en ligne le 31 décembre 2013, consulté le 03 mai 2019. URL : http://journals.openedition.org/ hommesmigrations/515; DOI : 10.4000/hommesmigrations.515 


\section{L'oubli au cour de la mémoire}

Le Museo Nacional de la Inmigración d'Argentine

Par Tamar Blickstein, doctorante au département d'anthropologie de l'université de Columbia, New York

La société argentine n'est pas si blanche que pourrait le laisser croire le Musée national de l'immigration de Buenos Aires. Nourrie des migrations européennes, elle l'est tout autant des migrations frontalières. Or l'absence des migrants non européens dans ce musée

témoigne de leur absence symbolique au sein d'une nation dont ils sont pourtant partie prenante. Par ses choix muséographiques qui réduisent au silence toute une partie de la population argentine, le musée met à jour le contenu problématique du creuset social argentin. 
Il n'y a pas d'absence de discours sur l'immigration en Argentine, pas de lacunes apparentes à combler par la cure du travail de mémoire. L'immigration est présente dans le discours officiel et non officiel, dans les journaux, les films, les manuels scolaires et la recherche universitaire. Les politiciens ont utilisé cette mémoire partagée dans leurs discours, les défenseurs des droits humains ont reconnu sa force, et les écrivains et les artistes trouvent encore en elle une source féconde d'inspiration. Selon une blague nationale éculée, Somos descendientes de los barcos, "Nous descendons tous des bateaux". Aussi l'exemple argentin illustre-t-il les limites du modèle mémoire-amnésie.

En outre, le mythe du melting-pot, le crisol de razas, est valorisé avec fierté et romantisme. La Constitution, qui date de 1853, déclare que l'immigration est une contribution bienvenue "au progrès et à l'industrie" de l'Argentine (articles 20 et 25). Aujourd'hui, les travailleurs migrants sont encore perçus comme les bâtisseurs du pays, qui ont apporté à la nation l'industrie, la technologie et des talents multiculturels. Il existe même une sorte d'Ellis Island argentin - lieu de mythe et de mémoire de l'immigration. C'est pourtant précisément ici, au Museo Nacional de la Inmigración (MNI), au cceur du bastion national de la mémoire migrante, que règne le plus profond silence sur l'immigration.

Ce musée concerne la représentation des migrants européens blancs, à l'exclusion flagrante de toute autre origine ethnique et nationale. Cette négligence persistante a fait l'objet d'un travail de terrain mené entre 2005 et 2006 à Buenos Aires. L'une des omissions les plus surprenantes du musée est celle des immigrés issus d'autres pays d'Amérique latine qui constituent aujourd'hui la grande majorité de la population d'origine étrangère. En dépit des incertitudes sur le pourcentage exact d'inmigrantes limitrofes ("immigrés de la frontière" issus de pays voisins) dans la population immigrée, on s'accorde à reconnaître leur prédominance.

ACTAS estime leur proportion à $60 \%{ }^{(1)}$, et Jachimowicz à environ $66 \% \%^{(2)}$, même si ces données, issues du bureau national du recensement ${ }^{(3)}$, ont été recueillies avant le krach de 2001, quand l'Argentine attirait encore un flux restreint mais non négligeable d'immigrés d'outre-Atlantique. Selon le Centro de Estudios Migratorios Latino Americanos de Buenos Aires, les flux post-crise argentine des pays riches ont baissé, faisant monter la proportion des limitrofes, surtout si l'on prend en compte les immigrés clandestins.

Souvent perçus à tort comme "les nouveaux immigrés", les migrants régionaux en Argentine sont déjà présents dans le premier recensement national de 1869 , comptant systématiquement pour environ $3 \%$ de la population totale selon des registres officiels. Il est ironique qu'une présence aussi constante dans les données nationales soit à ce point absente de cet hommage muséal. 


\section{L'immigration au cœur de l'histoire de l'Argentine}

Situé au sein de la Dirección de Migraciones (et donc dépendant du ministère de l'Intérieur), le bâtiment qui abrite ce musée d'État était autrefois destiné à accueillir et à nourrir des bateaux entiers d'immigrants venus d'outre-Atlantique ${ }^{(4)}$. La grande ironie, c'est que le musée a été inauguré en 2001, en plein krach économique, quand les descendants de ces mêmes migrants, en quête désespérée d'un passeport pour quitter le pays, se ruaient sur les bases de données du musée pour se trouver des ancêtres étrangers. Cette ironie prend des proportions théâtrales dès que l'on arrive sur le site du musée, qui n'occupe que l'un des bâtiments officiels dévolus à l'immigration. À côté se trouve le centre de naturalisation des étrangers - une sorte de préfecture où les immigrés des pays voisins font la queue dans l'angoisse pour renouveler leurs visas de travail. En cherchant les meilleurs moyens de traverser la redoutable autoroute devant la Dirección lors de mes trois visites au musée, j’ai souvent croisé d'autres étrangers, leurs papiers à la main. "Vous savez où se trouve le Museo Nacional de la Inmigración?' La douzaine de personnes quej'ai interrogées près de la Dirección n'avaient jamais entendu parler de cet endroit. Pour pénétrer dans la Dirección, le touriste fait la queue parmi les étrangers qui viennent faire renouveler leurs papiers. Comme une chorégraphie burlesque, l'appareil bureaucratique accomplit son tri entre "descendus des bateaux" et inmigrantes limitrofes. Une fois franchi le sas de sécurité, la voie bifurque. Les migrants tournent à gauche vers le centre de documentation où ils vont continuer à faire la queue pour leur visa. Le touriste ou l'Européen de deuxième génération va tout droit, traversant la vaste pelouse qui s'étend devant cet ancien hôtel pour migrants $s^{(5)}$.

Le MNI, lui, regarde la côte surplombant la vue du Río de la Plata. Cet estuaire qui sépare Buenos Aires de l'Atlantique est aussi le cordon ombilical reliant l'imaginaire argentin à l'Europe. L'édifice, marqué de taches d'humidité qui lui confèrent une certaine mélancolie, domine un port qui est une superposition d'inscriptions mémorielles. Ouvrant vers le nord-est, le bâtiment lui-même semble tourner le dos aux Amériques.

Les murs du musée sont couverts de panneaux d'information, largement composés par le fondateur du musée, le Dr Jorge Ochoa. Ils vont droit au cceur du sujet : l'histoire de la nation en tant qu'histoire de l'immigration. On trouve là nombre des éléments qui fondent Ellis Island, dont la louange amphigourique des travailleurs, des mineurs et des fermiers qui "abandonnèrent leur foyer, leur pays et leur langue" pour chercher un destin inconnu dans le Nouveau Monde.

Sur un autre mur, une grande carte des flux de migration en direction de l'Argentine, avec des flèches reliant divers endroits de l'Europe à Buenos Aires. Dans le hall suivant, le visiteur est accueilli par une douzaine de mannequins portant l'habit 
traditionnel de leur pays d'origine: des Italiens, des Basques et des Catalans, un couple d'Andalous et deux Suisses allemands, ainsi qu'un couple de juifs polonais. Parmi les "histoires de vie", on compte aussi un homme d'affaires turc.

Le musée cite la première Constitution argentine de 1853, qui instaure une nouvelle politique d'immigration invitant les migrants industrieux du monde à s'installer librement dans le pays (article 20). Mais il ne cite pas la préférence explicite de la Constitution pour les "migrants européens" (article 25) et sa politique de défense contre les infiltrations d'étrangers limitrophes, ni sa suspension par décret des droits constitutionnels en cas de "désordre" et de "commotion" aux frontières de la nation (article 23). Cette politique d'immigration fut au départ conçue par le plus libéral des pères fondateurs, qui reste à ce jour un point de référence culturel dans toute l'Amérique latine: Domingo F. Sarmiento (1811-1888), écrivain, penseur et politicien, qui fut le septième président d'Argentine entre 1868 et 1874 . Aujourd'hui, son buste orne les nombreuses écoles et bibliothèques nationales dont il fut l'ardent promoteur, et ses cuvres sont inscrites au programme des écoles. Considérant le poids symbolique de l'héritage de Sarmiento, sa contribution à la mythologie nationale de l'immigration et l'importance historique de ses écrits dans la formation de l'identité nationale, son ceuvre présente ici un intérêt sociologique. Il est bon en conséquence de nous pencher sur son texte le plus lu, et cité en référence dans les écoles argentines : Facundo: Civilization y barbarie ${ }^{(6)}$.

\section{Une identité nationale fantasmée}

Souvent considéré comme le premier essai littéraire d'Amérique latine, Facundo est à la fois un roman, un pamphlet politique, un essai ethnographique et un récit de voyage. Exilé pour avoir combattu la direction autoritaire de Juan Manuel de Rosas, le gouverneur de Buenos Aires de 1829 à 1840, Sarmiento se lamente sur la destinée de sa jeune nation avec une angoisse lyrique propre au romantisme qui le fascinait. Pour lui, toute la problématique de l'Argentine se résume à l'opposition manichéenne entre culture et nature, entre civilisation et barbarie : "Être ou ne pas être un sauvage, telle est la question ${ }^{(7)}$." Le problème pour Sarmiento est profondément politique, et sa quête d'identité peut se lire comme une allégorie de la nation émergente ${ }^{(8)}$.

Sarmiento est une figure paradoxale. Autodidacte issu d'un humble milieu rural, c'était un humaniste qui croyait en l'éducation universelle et défendait les droits des pauvres migrants (européens) contre la répression xénophobe et oligarchique de l'époque. Mais il lança aussi une série de rageuses campagnes génocidaires contre les peuples indigènes, et chercha à éradiquer toutes les lignées, cultures et langues portant des traces non européennes. 
Dans Facundo, Sarmiento exprime la dialectique entre civilisation européenne et barbarie ibéro-américaine comme un combat personnel et corporel entre son propre instinct nomade et son goût fervent, quasiment religieux, pour l'Europe des Lumières. La question le conduira en exil au Chili, où elle continuera à le hanter sans trêve, sourdant de ses analyses politiques et ethnographiques, l'accompagnant jusqu'au fin fond de la pampa où il affronte le séduisant cauchemar du gaucho, d'où son livre tire son titre. Ce gaucho, Facundo, représente l'esprit menaçant du mestizaje qui plane sur “les plaines désertes" de l'Argentine, jusqu'à se confondre avec elles. Ce métissage n'est pas purement phénotypique, mais également social, politique, culturel et linguistique. Le rapport complexe de Sarmiento au gaucho n'est pas étranger au projet de blanchiment dont on retrouve l'écho dans les panneaux d'affichage du MNI.Si l'Ellis Island argentin semble au premier abord un signe multiculturel d'intégration heureuse, un examen plus approfondi des

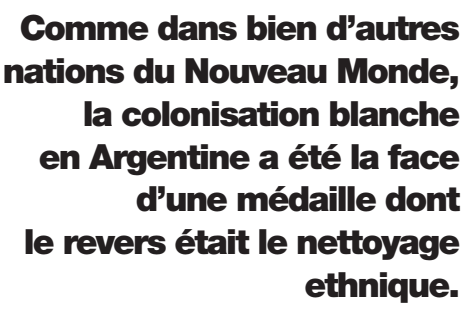

histoires nationales dévoile souvent une autre vérité.

Malgré la fervente croyance de Sarmiento dans la philosophie darwiniste positiviste et sociale de son époque, ses écrits révèlent ses doutes sur "l'inévitable triomphe des Blancs dans le combat mondial ${ }^{9}$ ". La progression des Blancs en Argentine risquait d'être sapée par le métissage avec des races "non civilisables"

comme les Guarani - un mélange contaminé, se lamentait Sarmiento, qui au lieu de conduire à la stérilité entraînait une abondante fécondité portant le pire des deux races. ${ }^{(10)}$ Le gaucho représente cette redoutable alchimie des sangs, tout en étant d'une déconcertante familiarité. Comme Sarmiento lui-même, il est un pur produit du monde rural, un esprit férocement indépendant, un vagabond suivant sa propre voie, et un autodidacte dans son domaine. En 1919, Freud définissait l'Unheimliche, l'inquiétante étrangeté (ou, littéralement, le non-foyer), comme une dissonance cognitive ou une répulsion obsessionnelle face à quelque chose qui semble étranger et pourtant étrangement familier ${ }^{(11)}$. Le mestizaje du gaucho est un mélange pervers entre l'esprit familier et humain du criollo d'ascendance espagnole et le corps étranger inhumain du Sud-Américain indigène. C'est dans ce miroir Unheimliche que Sarmiento plonge le regard, pour ne saisir qu'une fugitive vision de la future nation, déchirée entre l'héritage du despotisme espagnol et la sauvagerie nomade. Comme dans bien d'autres nations du Nouveau Monde, la colonisation blanche en Argentine a été la face d'une médaille dont le revers était le nettoyage ethnique. Mais celui-ci n'a pas été réalisé d'abord par des campagnes génocidaires, comme on le croit souvent. 


\section{Un métissage problématique}

Contre le métissage, Sarmiento a inventé le melting-pot "blanchissant" le plus influent d'Amérique latine. Le mythe pluraliste des origines, joint à une politique d'immigration apparemment ouverte, faisait partie d'un projet eugéniste national pour résoudre "le problème de la barbarie". Le blanchiment de l'Argentine fut interprété comme un grand succès par les contemporains de Sarmiento, et un modèle que d'autres nations américaines et caribéennes tentèrent d'imiter ${ }^{(12)}$. En 1860, Buenos Aires fut inondée d'Italiens, d'Espagnols, de Basques et de quelques groupes plus restreints de juifs d'Europe de l'Est et de "Turcs"(terme européanisant pour désigner les musulmans et les Arabes en général). Leur nombre surpassait celui des nationaux dans la capitale: il atteignit 2,5 millions entre le premier recensement national de 1869 et celui de 1914, ce qui conduisit à la construction de l'Hotel de Migrantes en 1906. Certes, ces immigrés n'étaient pas d'ascendance anglo-saxonne comme Sarmiento, Alberdi, Bunges, Ingenieros et d'autres auraient pu l'espérer, mais la plupart étaient européens. Ces origines diverses se fondraient dans l'éducation républicaine universelle telle que la voyait Sarmiento, un modèle de crisol de razas centré sur la capitale et effaçant symboliquement la présence de tous les non-Européens.

Mais les provinces argentines racontent une tout autre histoire. À partir des années trente, l'immigration européenne s'épuise, tandis que les migrants frontaliers continuent à entrer en masse en Argentine. Marginalisés et rendus invisibles dans l'imaginaire national centré sur la capitale, ces migrants et autres non-Blancs, dont les Afro-Argentins, furent "exclus de la définition symbolique de la nation ${ }^{(13)}$ ". Pourtant, cette "non-présence" était une réalité constante dans les provinces. En outre, la brutale Conquête du désert (1821-1899) envisagée par Sarmiento et menée par le général Julio A. Roca fut loin d'être la réussite qu'ils imaginaient. Plus de la moitié des peuples indigènes survécurent aux campagnes, et seuls $6 \%$ furent tués directement au combat $t^{(14)}$. Le plus souvent, la "destruction" de ces peuples fut en fait obtenue par des moyens moins coûteux et plus utiles aux élites locales et aux corps gouvernementaux. Les survivants de la Conquête du désert furent soumis à des politiques de "civilisation", d'"assimilation" et de "détribalisation" consistant pour l'essentiel en servitude domestique et en concubinage forcé pour les femmes, et pour les hommes en exercice militaire et travail obligatoire forcé dans les champs de canne à sucre et les vignobles en manque de main-d'ceuvre ${ }^{(15)}$. Ces mêmes secteurs agricoles recrutaient souvent des travailleurs saisonniers à bas coût venus du Paraguay, de Bolivie, du Chili, de l'Uruguay et du Brésil, maintenant ainsi un flux constant de migrants frontaliers tout au long de l'histoire de l'Argentine. La confluence entre ces deux sources de travail donna naissance à une classe inférieure métissée qui allait devenir de plus en plus visible dans 
les agglomérations urbaines. Contre une capitale se percevant comme un crizol de razas tout européen, les provinces étaient bien plus hétérogènes - une réalité absente de la narration nationale majeure et discrètement omise dans les textes d'histoire, les manuels scolaires, les statistiques et les mémoriaux comme le MNI. Là encore, l'oubli national argentin tient essentiellement à la place qu'il accorde à la mémoire nationale de l'immigration au sein d'une mémoire nationale hégémonique.

\section{Clivages symboliques et clivages sociaux dans la société argentine}

Létrange silence qui règne dans les corridors du MNI n'a nullement été rompu par la "résolution" de Sarmiento. Cette invisibilité historique s'est révélée de plus en plus insoutenable, même au sein de la capitale européocentrée. L'industrialisation du $\mathrm{XX}^{\mathrm{e}}$ siècle a généré des vagues d'urbanisation, ouvrant les portes de Buenos Aires et d'autres grandes villes aux pauvres des provinces. C'est sur cette classe inférieure du mestizo issue de ces politiques d'assimilation que s'est ensuite appuyé Juan Domingo Peron de 1943 jusqu'au milieu des années cinquante, quand il devint le fer de lance symbolique du premier mouvement politique populaire de masse avant d'être élu président. Le terme de cabecitas negras ("petites têtes noires"), introduit au milieu des années quarante par les élites blanches pour déligitimer les péronistes, révèle combien la politique de classe s'était racialisée dans la capitale. Peron et sa femme Eva allaient se réapproprier cette expression comme un terme amical désignant les mestizos ruraux recrutés pour travailler dans les villes et venant consolider leur soutien ouvrier. Au cours de nombreux renversements politiques et coups d'État militaires, les villas (quartiers populaires d'invasions) furent souvent publiquement condamnées et même mises "hors la loi", avec des morts et des évictions, des déplacements de masse et des regroupements dans ce qui allait finalement devenir de nouvelles villas ${ }^{(16)}$.

Comme dans d'autres grands pays hôtes, la création en Argentine d'un musée national consacré à un thème aussi controversé que l'immigration s'est inscrit dans ce que Bourdieu appelle "le champ du pouvoir ${ }^{(17) "}$. À ce titre, le musée était soumis aux intérêts en concurrence et aux héritages historiques du pouvoir. Il représente la victoire silencieuse d'un vieux consensus hégémonique qui persiste dans le présent. Ce n'est pas un hasard si le musée a été officiellement approuvé et financé sous le régime néo-libéral du président Carlos Menem, dans les années quatre-vingt-dix. Considéré comme une longue période de transition démocratique après une brutale dictature militaire (1976-1983), le règne de Menem fut aussi marqué par une rapide polarisation des différences de classe, et par une explosion des villas urbaines où la 
première et la seconde générations de migrants argentins des provinces côtoyaient les inmigrantes limítrofes ${ }^{(18)}$. Les anciens résidents des villas déplacés pendant la dictature rentrèrent à Buenos Aires en quête de travail, suivis de travailleurs ruraux au chômage du fait de récentes dénationalisations. À mesure que les différences de classe se racialisaient au centre des villes, des insultes comme bolita (péjoratif pour Bolivien), negro et cabecita negra devinrent interchangeables, comme elles le sont encore aujourd'hui ${ }^{(19)}$.

Le MNI est l'un de ces coûteux projets de développement urbain conçus pour faire de Buenos Aires une brillante capitale du premier monde, rappelant la "lumineuse et accueillante époque" où les Européens se pressaient bruyamment sur les rives du "grenier du monde" sud-américain ${ }^{(20)}$. Le musée, définissant sa mission, déclarait fièrement que "sa représentation muséale [de l'immigration] devait relier notre histoire à celle du reste du monde", du fait des "caractéristiques distinctives qu'a eues l'immigration en Argentine ${ }^{(21)}$. Ce crisol particulier était "le lien au monde" de l'Argentine, son billet symbolique de sortie des Amériques et sa légitimité pour intégrer "le Nouvel Ordre du monde". Le creuset européen de Sarmiento allait enfin accomplir sa destinée de progrès en rendant à l'Argentine son ancien rang sur la scène mondiale. Si le musée n'était pas conçu comme un instrument politique en soi, sa réalisation comme projet gouvernemental reflétait les enjeux socio-économiques de l'européanité, et le déni opiniâtre de légitimité politique à "d'autres" immigrés et Argentins au sein d'un ethnoscape urbain radicalement changeant ${ }^{(22)}$. Si le musée expose une Argentine en phase avec le " progrès du premier monde", l'absence de migrants non européens en son sein entre en résonance avec un ancien et profond silence national.

\section{La construction du silence des migrants non européens}

Cette immigration rendue silencieuse a enfanté des traces vivantes, qui se manifestent par les diverses formes d'exclusion et d'invisibilité que subissent les "migrants de la frontière" dans leur vie quotidienne, comme dans les bureaux de l'immigration voisins du musée MNI. Cette invisibilité porte un long et complexe héritage, qui a instrumentalisé les stéréotypes racistes faisant des peuples indigènes des "fatalistes silencieux" et a qualifié la terre qu'ils habitaient de "stérile".

De nos jours, les deux premières générations de migrants issus des pays voisins comme la Bolivie, de même que les non-migrants qui leur ressemblent, sont stéréotypés sous le terme de callados (silencieux) au travail comme à l'école. Ce silence est en général interprété comme un obstacle au savoir, au progrès et à l'efficacité, même par des 
enseignants bien intentionnés ${ }^{(23)}$. Pour Novaro $^{(24)}$, ce silence a été réifié en un véritable phénomène social, "incarné" et "acté" par de jeunes migrants de la deuxième et de la troisième générations dans les écoles publiques de Buenos Aires. Cette perception fantasmatique d'une présence sans voix a été confirmée par mes propres recherches. La directrice d'une école publique de Bajo Flores, une banlieue appauvrie de Buenos Aires, me confiait que le silence est un véritable problème dans son école pleine de migrants, problème qu'elle qualifie de "caractéristique" des enfants les plus indigènes. L'une de ses institutrices ajoutait qu'enseigner à un enfant qui ne parle pas est une expérience frustrante, parce qu'on se demande "s'il y a quelqu'un là, s'ils existent vraiment". Un corps sans voix dans la salle de classe pose des problèmes évidents aux pratiques d'assimilation.

à la perception fausse mais largement répandue que le nombre de migrants frontaliers s'est drastiquement accru au cours des dernières décennies.
En outre, la légende d'un Buenos Aires européen et blanc a conduit à une perception exagérée du nombre de migrants, puisque toute apparence non européenne est censée venir d'ailleurs ${ }^{(25)}$. L'un des journaux les plus xénophobes de la dernière décennie diabolisait les migrants en parlant d' "une invasion silencieuse ${ }^{(26) "}$. Cette "invasion" semble d'autant plus invasive qu'elle est "silencieuse", menace rendue plus menaçante encore par son caractère impondérable. Des anthropologues ont observé une confusion discursive entre populations migrantes et argentine dans des espaces et des événements publics tels que les matchs de football. Selon Alejandro Grimson, les migrants boliviens sont socialement considérés comme des cabecitas negras, tandis que les citoyens argentins ayant une ascendance andine ou une parenté bolivienne sont socialement considérés comme des Boliviens ${ }^{(27)}$. Cette confusion racialisée entre migrants nationaux et étrangers conduit à la perception fausse mais largement répandue que le nombre de migrants frontaliers s'est drastiquement accru au cours des dernières décennies. Cela peut aussi expliquer pourquoi La Primera dépeignait les migrants comme une sorte de marée fantomatique, une "invasion silencieuse" pénétrant subrepticement le marché du travail et les taudis de Buenos Aires. Comme les peuples indigènes des provinces du Nord-Est et du Sud, et comme le gaucho "métissé" qui a hanté Sarmiento jusqu’à la folie, ces migrants et nonmigrants sont perçus comme des fantômes d'une sphère intangible mais répugnante qui a toujours plané obscurément sur les faubourgs de l'imaginaire argentin. Ils sont une non-entité de masse secrète et sans voix, une présence déconcertante, imperceptible, étrangère tout en n'étant que trop familière. 


\section{Les immigrants limitrophes: une présence fantomatique}

C'est précisément cette logique qu'invoque l'administration du MNI pour justifier l'exclusion des migrants latino-américains de sa narration. Dans un entretien de 2005, feu le fondateur du musée expliquait qu'ils n'étaient pas et ne seraient pas présents dans le musée parce que les Argentins ne les considèrent pas vraiment comme des migrants, beaucoup de nationaux argentins des provinces du Nord ressemblant ("gualitos") aux migrants boliviens ou paraguayens. Il citait leur nature inexpressive comme un signe de leur désir de rester absents de l'histoire du musée. Enfin, il pointait leur ascendance visiblement non européenne comme le facteur surdéterminant de leur identification : à la fin de la journée, ils retournent à leur origine indigène, "se vuelven indios".

"Ils /les inmigrantes limítrofes]sont par nature des gens discrets, qui n'aiment pas se dévoiler (...). Mais curieusement, le jour de la Fiesta de la Virgen, ils jouent de la quena /guitare andine traditionnelle], ils mettent des masques et ils dansent. Ils redeviennent indiens ${ }^{(28)}$ !' Avoir des ancêtres non européens semble vous disqualifier pour le statut de migrant, au-delà du régime des frontières nationales. Selon cette logique, un "immigré" est par définition européen, un "citoyen” du melting-pot destiné au progrès. En revanche, tout lignage indigène pousse le migrant latino-américain hors de la citoyenneté symbolique, le renvoyant à l'impasse silencieuse de la terre. Le mot "volverse" est significatif ici. Il signifie "redevenir", "revenir à un point antérieur". Son cheminement n'est pas linéaire, mais circulaire et régressif. Sa progression défait l'étape précédente, suggérant un sentiment du temps circulaire plutôt qu'historique. Le directeur du MNI n'avait peut-être pas de telles intentions, mais le mot qu'il emploie reflète les limites de la citoyenneté exprimées par le musée, et le favoritisme de la Constitution. Aux yeux de la nation, tous les migrants ne sont pas nés égaux. La frontière entre citoyen et étranger est bâtie sur des lignes paradoxales. Comme l'État-nation de Sarmiento, le musée doit faire taire les Amériques pour pouvoir exister comme européen ; il doit éradiquer cette "non-présence" de sa mémoire pour affirmer publiquement une fragile citoyenneté dans l'ordre du monde. Aujourd'hui, alors que Buenos Aires déborde de ces visages que l'histoire argentine prétend avoir oblitérés, les élites de la capitale doivent se demander pourquoi "celui qui a disparu semble toujours être là", et si "le cadavre peut-être n'est pas mort, aussi simplement mort que la conjuration s'efforce de nous pousser faussement à le croire ${ }^{(29) !}$. L'Histoire nous le dira. Alors que le musée tente de revisiter l'esprit républicain de Sarmiento, il peut bien finir par invoquer ces mêmes fantômes nationaux que Sarmiento a tenté autrefois d'exorciser.

Traduit de l'anglais par Françoise Bouillot 


\section{Notes}

1. ACTAS, Maria Skoczek, Guadalupe Vargas Montero (dir.), Migraciones laborales contemporáneas y su rol en el desarrollo local y regional en América latina y Europa, ACTAS Latino americanas de Varsovia, $49^{\circ}$ Congreso Internacional de Americanistas, Quito, 7-11 juillet 1997.

2. Maia Jachimowicz, "Argentina : a new era of migration and migration Policy", in Migration Information Source, Washington D. C., Migration Policy Institute, 2006. http://www.migrationinformation.org/Profiles/display.cfm? ID=374.

3. INDEC, Población limítrofe por año de llegada a Argentina. 2002-2003, 2004. http://www.indec.mecon.ar/default.htm.

4. Le Museo Nacional de la Inmigración dépend totalement de la Dirección de Migraciones. Malgré son nom, il n'a aucun statut officiel, étant considéré comme un simple "programme" (Programa complejo museo de la inmigración). À l'époque, il n'avait pas de directeur, mais un "coordinator" embauché par le Director de Migraciones.

5. Entretiens avec des "immigrantes limitrofes" faisant la queue à la Dirección lors de mes trois visites, ainsi qu'avec d'autres immigrés dans Buenos Aires.

6. Domingo Faustino Sarmiento, Facundo. Civilización y Barbarie, Havana, Casa de las Américas, Colección Literatura Latinoamericana, $1982[1845]$.

7. Ibid.p. 22.

8. Fredric Jameson, "Third-world literature in the era of multinational capitalism", in Social Text, n 15, 1986, pp. 65-88.

9. Aline Helg, "Race in Argentina and Cuba, 1880-1930. Theory, policies, and popular reaction", in Richard Graham (dir.), The Idea of Race in Latin America, Austin, University of Texas Press, 1990, p. 40.

10. Le gaucho de Sarmiento est intimement lié au sauvage "fatalisme" censé caractériser l'indigène. S'il plaçait tous ces peuples au bas de l'échelle évolutionnaire, Sarmiento jugeait les Guaraní et les Mapuche d'Argentine particulièrement proches des animaux, sorte de race préhistorique impossible à civiliser (Helg 1990).

11. Sigmund Freud, L'Inquiétante étrangeté et autres essais, Paris, Gallimard, 1988.

12. Aline Helg, "Race in Argentina and Cuba, 1880-1930. Theory, policies, and popular reaction", op. cit.

13. Monica Quijada, "La question indienne", in Cahiers Internationaux de Sociologie, vol. $45, n^{\circ} 105,1998$, p. 306 ; George Reid Andrews, The Afro-Argentines of Buenos Aires, 1800-1900, Madison, University of Wisconsin Press, 1980.

14. Monica Quijada, "La question indienne", op. cit., p. 323.

15. Claudia Briones, Walter Delrio, "Patria sí, colonias también. Estrategias diferenciadas de radicación de indígenas en Pampa y Patagonia (1885-1900)", in Ana Teruel, Monica Lacarrieu, Omar Jerez (dir.), Fronteras, Ciudades y Estados, Córdoba, Alción Editora, 2002, pp. 45-78 ; Enrique Mases, "Estado y cuestión indígena en la Argentina finisecular. La incorporación de los indios sometidos", in Índice : Revista de Ciencias Sociales, vol. 35, 2001, pp.189-207.

16. Emanuela Guano, "The denial of citizenship : 'barbaric' Buenos Aires and the middle-class imaginary", in City \& Society, vol. 16, n 1, 2004, pp. 69-97.

17. Pierre Bourdieu, La Noblesse d'État, Paris, Minuit, 1989.

18. Emanuela Guano, "The denial of citizenship : "barbaric' Buenos Aires and the middle-class imaginary", op. cit.

19. Alejandro Grimson, "Nuevas xenofobias, nuevas políticas étnicas en la Argentina", in Alejandro Grimson,

Elizabeth Jelin (dir.), Migraciones regionales hacia la Argentina : diferencia, desigualdad, derechos, Buenos Aires, Prometeo Libros, 2006, pp. 69-97.

20. Dirección Nacional de Migraciones, Proyecto: Museo Nacional de la Inmigración, Buenos Aires, 2000, p. 8.

21. Ibid., p. 6.

22. Arjun Appadurai, "Global ethnoscapes. Notes and queries for a transnational anthropology", in Modernity at Large: Cultural Dimensions of Globalization. Minneapolis, University of Minnesota Press, 1996, pp. 48-65. [Après le colonialisme. Les conséquences culturelles de la globalisation, Paris, Payot, 2003.]

23. Mariana Beheran, "Identificaciones étnico-nacionales de jóvenes inmigrantes en una escuela pública de nivel medio de la ciudad de Buenos Aires", in Congreso Argentino de Antropología Social, Misiones, Argentine, 7 août 2008.

24. Gabriela Novaro, Laureano Borton, Maria Laura Diez et Ana Carolina Hecht, "Sonidos del silencio, voces silenciadas. Niños indígenas y migrantes en escuelas de Buenos Aires", in Revista Mexicana de Investigación Educativa, vol. 38, $\mathrm{n}^{\circ} 15$, Mexico, 2008, pp. 173-201.

25. Emanuela Guano, "The denial of citizenship : 'barbaric' Buenos Aires and the middle-class imaginary", op. cit.

26. Titre d'un article de la revue de droite La Primera, 2000.

27. Alejandro Grimson, "Nuevas xenofobias, nuevas políticas étnicas en la Argentina", op. cit., p. 78.

28. Entretien avec J. Ochoa, directeur du MNI, Buenos Aires, décembre 2005.

29. Jacques Derrida, Spectres de Marx, Paris, Galilée, 1993, p. 97. 